\title{
Editorial: Crop Biofortification for Food Security in Developing Countries
}

\author{
Abdul Wakeel ${ }^{1}$ and Maryke T. Labuschagne ${ }^{2 *}$ \\ ${ }^{1}$ Institute of Soil and Environmental Sciences, University of Agriculture Faisalabad, Faisalabad, Pakistan, ${ }^{2}$ Department of \\ Plant Sciences, University of the Free State, Bloemfontein, South Africa
}

Keywords: biofortification, provitamin A, iron, zinc, malnutrition

Editorial on the Research Topic

Crop Biofortification for Food Security in Developing Countries

Biofortification is the nutritional enhancement of crops through agronomic practices, conventional plant breeding practices, and/or genetic modification. Communities in developing countries often rely on staple crops like maize, cassava, wheat, rice, and beans to supply most of their energy needs, and biofortification of these staples will therefore benefit such communities. Biofortification could have long-term benefits in groups that do not have access to other micronutrient interventions. Essential macro- and micronutrient deficiencies in human diets pose serious health challenges in developing countries. Several interventions have been used to increase dietary intake of nutrients, such as supplementation, dietary diversification, and crop biofortification. Genetic biofortification would be the most appropriate, cost-effective and sustainable intervention in minimizing nutrient deficiencies in communities subsisting predominantly on a few staples. Plant breeding and transgenics hold great promise for contributing to improvement of the nutritional status of staple crops across the world. The most common deficiencies in developing countries are vitamin A, folate, iron, iodine, and zinc, which can be addressed by biofortification.

Several efficacy studies have demonstrated that biofortification of staple crops can contribute to alleviating micronutrient malnutrition or "hidden hunger" among poor resource populations, especially in developing countries. There is dire need for innovative strategies to combat "hidden hunger" making biofortification more effective, especially for developing countries. This issue is composed of original research articles from discovery and application of novel tools or techniques to adoption of biofortified crops. Review articles grasping up to date research and assessments of social, economic, or nutritional impacts of biofortified crops have also been covered in this issue.

Natesan et al. evaluated different Indian maize landraces stressing that newly identified $\beta$ carotene-rich maize landraces can effectively be utilized in the breeding programs to enhance the $\beta$-carotene content in maize to combat the malnutrition enhancing biofortification process. Zia et al. described the biofortification of $\mathrm{Zn}$ in wheat through agronomy and breeding. They evaluated the high-Zn variety of wheat (Zincol-2016) and $\mathrm{Zn}$ fertilizers improving $\mathrm{Zn}$ concentration in grains under field conditions and concluded that environment and management affect the performance of "high-Zn" wheat varieties and there is need to design studies carefully to observe the realistic impact on human diet and nutrition. Malik and Maqbool stressed the need of biofortification to eradicate hunger by 2030, considering the UN's sustainable development goals. They focused on the national nutritional survey conducted in 2011 in Pakistan which indicated that more than $50 \%$ of the population face food insecurity due to acute micronutrient deficiency resulting in several disorders, mainly in the female population. Reviewing various studies which used transgenic 
approaches, it has been stressed that worldwide efforts made to improve the nutritional status of the human population are based on numerous biotechnological advancements.

Beebe reviewed the research accomplished on improving $\mathrm{Fe}$ content in common bean (Phaseolus vulgaris L.) with special reference to African countries. In a bio-efficacy trial among teenage women of Rwanda, Fe biofortified beans improved $\mathrm{Fe}$ content and boosted brain function and cognitive ability of the female participants. Nevertheless, it is mentioned that the progress in breeding is very slow, which might be due to homeostatic mechanisms of $\mathrm{Fe}$ and $\mathrm{Zn}$ uptake. It is also contended that use of sister species evolved in Fe-deficient environments can enhance the success of biofortification. Improving the bioavailability of $\mathrm{Fe}$ through future breeding programs may require further consideration.

Akinsola et al. studied the bioavailability of provitamin A from a maize snack (Kokoro) in south western Nigeria. It was reported that conventional breeding improves the provitamin A in indigenous white maize used to produce Kokoro. The research has shown that nutritious Kokoro with a high carotenoid content can be made from provitamin A maize cultivars, in contrast to commonly available Kokoro deficient in carotenoids. However, increase in bioavailable provitamin A content was variable in different genotypes.

Rathan et al. revealed from a study using 94 wheat genotypes enriched with $\mathrm{Fe}$ and $\mathrm{Zn}$ that biofortification does not affect the quality of wheat considering processing quality and glutenin profile. They also found that grain $\mathrm{Fe}$ and $\mathrm{Zn}$ contents are only linked to grain protein content without affecting other quality traits.

Rehman et al. reviewed the progress on agronomic biofortification of cereals with $\mathrm{Zn}$ in Pakistan. It was concluded that various agronomic strategies for $\mathrm{Zn}$ biofortification caused significant improvement in grain $\mathrm{Zn}$ concentration and improved grain yield from $\mathrm{Zn}$ enriched seeds under abiotic stresses. Agronomic interventions can reduce the disease burden due to $\mathrm{Zn}$ deficiency, however, there is need for government policies for farmer awareness programs, subsidies to farmers, and promotion of $\mathrm{Zn}$ biofortified foods in the country.

Mwanga et al. critically analyzed the work progress on Vitamin $\mathrm{A}, \mathrm{Fe}$, and $\mathrm{Zn}$ biofortification considering drought tolerance and disease tolerance of sweet potato. It was concluded that accelerated breeding programs have done superbly in Africa to develop varieties enriched with above mentioned traits in sweet potato, however there is need to further accelerate the breeding program considering further required traits to improve human nutrition.

The papers in this collection show the progress made in recent years in crop biofortification, and its application in the developing countries, especially. It reflects the success of $\mathrm{Zn}$ biofortification in wheat, which has been brought about largely by the work of HarvestPlus and CIMMYT in Mexico. It also reflects the successful provitamin A biofortification of crops such as maize and sweet potato. The increasing importance and application of genetic engineering in biofortification are also highlighted. In all it is clear that biofortification will be an inherent part of future breeding strategies particularly in developing countries, and that it is the most sustainable way to improve nutritional status in poor communities. As such, crop biofortification can contribute to food security in the developing world.

\section{AUTHOR CONTRIBUTIONS}

All authors listed have made a substantial, direct and intellectual contribution to the work, and approved it for publication.

Conflict of Interest: The authors declare that the research was conducted in the absence of any commercial or financial relationships that could be construed as a potential conflict of interest.

Publisher's Note: All claims expressed in this article are solely those of the authors and do not necessarily represent those of their affiliated organizations, or those of the publisher, the editors and the reviewers. Any product that may be evaluated in this article, or claim that may be made by its manufacturer, is not guaranteed or endorsed by the publisher.

Copyright (C) 2021 Wakeel and Labuschagne. This is an open-access article distributed under the terms of the Creative Commons Attribution License (CC BY). The use, distribution or reproduction in other forums is permitted, provided the original author(s) and the copyright owner(s) are credited and that the original publication in this journal is cited, in accordance with accepted academic practice. No use, distribution or reproduction is permitted which does not comply with these terms. 